\title{
EMPENYORAMENT DE JOIES I OBJECTES DEL REI JOAN I, FET PER LA REINA MARIA DE LUNA (1396)
}

\section{Maria Vilar Bonet}

Durant l'Edat Mitjana, la penúria constant de la Tresoreria Reial obligava, de vegades, els Sobirans a empenyorar les joies, com a garantia d'una quantitat manllevada per atendre les necessitats de la Corona.

De tals prestacions en penyora fetes per la reialesa, se'n podrien aportar moltes mostres, però ara, en el present treball, ens referirem a un empenyorament d'objectes del rei Joan I, fet per la reina Maria de Luna.

El 19 de maig de 1396, moria Joan I. No deixava fills mascles i, per tant, la successió del Regne corresponia al seu germà Marti, duc de Montblanc.

Catalunya reconegué immediatament com a sobirana la duquessa de Montblanc, que amb prudència i sagacitat es disposà, des del primer moment, a resoldre els problemes de la Corona.

Amb el fi de constrènyer els perills que envoltaven el Regne, convocà el Parlament, que prengué l'acord de resistir amb les armes les pretensions a la Corona del comte de Foix, que es disposava a envair el Principat.

En aquest Parlament de 1396 s'acordà, també, que el General de Catalunya armaria dues galeres, que amb una ambaixada, elegida i nomenada pel mateix Parlament, anirien a Sicilia i portarien al rei Martí 40.000 florins d'or d'Aragó per socórrer les ne- 
cessitats de les tropes que eren al seu servei en aquell Regne, atendre el que li fos necessari i retornar de Sicília quem adventum multum necessarium et utilem rei publice principatus Cathalonie reputabant.

Tal com s'havia concordat en el Parlament, el General armà les dues galeres i els Diputats entregaren als ambaixadors els 40.000 florins, que posaren en mans del rei Martí.

La reina Maria havia firmat als Diputats un debitori per valor de $\mathbf{4 0 . 0 0 0}$ florins, i com a garantia els va empenyorar objectes del rei Joan, «en aquesta manera: que les reliquies, robes e joyes que foren del senyor Rey en Johan, sien posades en penyora per la senyora Reyna en poder dels Deputats; e mes la dita senyora Reyna mete en poder dels Deputats, per habundant penyora, la vila de Montblanch ab tots drets d'aquella)' ${ }^{\text {. }}$

Tres anys i mig després, en un document datat a Saragossa el 25 de novembre de 1399 , es descriuen amb tota minuciositat els objectes empenyorats per la reina Maria de Luna i que el rei Martí confessa que ha recuperat ${ }^{2}$.

La intensitat descriptiva de la relació d'objectes ens permet conèixer amb detall cadascuna de les peces referides i reconstruir una part de l'esplendor artística a l'Edat Mitjana, la tècnica i el gènere de vida de l'època a què pertany. Tant per a l'historiador com per a l'arqueòleg, resulta altament interessant, sobretot perquè molts objectes han desaparegut, segurament fosos per convertir-los en altres de nous, de diferent forma i ús, a mesura que el gust anava canviant.

El conjunt de material que aquest document inèdit ens ofereix és d'un efecte enlluernador. Hi figura tota una sèrie d'objectes litúrgics: creus, reliquiaris, imatges, canelobres; una vaixella; espases. Fets d'or, plata, ivori, cristall, ambre, marbre, jaspi. Adornats amb perles, maragdes, balaixos, safirs, diamants, corall i amb una gran profusió d'esmalts. En destaquem la diversitat de relíquies i també la varietat de teles, entre les quals hi

Cortes de los antiguos reinos de Aragón y Valencia y Principado de Cataluña, Real Academia de la Historia, Madrid 1901, Tomo IV, p. 297.

${ }^{2}$ ACA, Reg. 2315, fols. 19 r.-26 v. 
ha atzeitoní, bocaram, cendat, cotó, domàs, lli, savastre, seda i vellut. Tot amb una gran riquesa de colors, blanc, vermell, rogicler, groc, verd, blau, morat, negre.

En el present treball transcrivim la relació integra dels objectes descrits en aquest document de 1399 . Hem numerat les diverses partides per a una major claredat, i afegim, després, un index dels objectes $i$ elements que ens han semblat més interessants.

[ 1.] «Primo .I. confiter ab son peu levadiç d'argent daurat dins e de fora, e lo dit peu a part de fora e en mig del confiter ha .I. armalt en que es lo timbre del dit senyor; entorn del dit confiter ha tres senyals, la .I. reyal, c l'altre d'Aragó, e l'altre la creu de Sent Jordi ab .IIII. testes de moros, e gravat, per les ores fet ab rosetes petites, e tot entorn ab garlanda de fulles, e lo cano obrat de maçoneria ab senyals; e lo peu son VI. gallons en que ha los dits tres senyals, en los altres gallosn ha tres corones. E pesa a march de Barchinona. XXII. marchs,.$I^{a}$. onza. Hay .I. trocet del peu separat ab .III. muronets.

[ 2.] Item .I. peu e sobrecop d'argent sobredaurat, qui era fet per copa de vidre. Qui pesa .III. onzes, .III. quarts e mig.

[ 3.] Item.$I^{2}$. caldereta d'argent blanch ab les ores daurades. E .I. salpasser d'argent. Pesa .I. march, .VI. onzes, .I. quart.

[ 4.] Item .I. bassi d'argent blanch per fer la barba. Qui pesa .IIII. marchs, .VII. onzes, mige.

[ 5.] Item . $\mathrm{I}^{\mathrm{a}}$. correge d'or $\mathrm{ab}$ parxe de seda negre, $\mathrm{ab}$.I. listo de seda vermella al mig, ab caps e .VII. platons. Pesa .IIII. onzes, .III. quarts, mig.

[ 6.] Item .I. coltell fet a manera de basalart, lo qual es guarnit d'or ab lo bocal, e al mig on esta lo ganivet e guaspe ab la empresa de la corona, ab manech, croera de ferra e lauto daurat, ab lo foure negre, e la correge de sanastre d'or, cap, e mosqueta, e .V. platons d'or.

[ 7.] Item .I. basalart ab caps, croera de I[a] uto, lo bocal, e la on esta lo ganivet e guaspe d'or, ab la dita divisa, ab una corregeta de sede negre en que penge lo dit besalart, cap, mosqueta e VIIII. platons d'or, la bayne de cuyr negre.

[ 8.] Item .I. altre basalart guarnit d'argent, lo bocal e al mig, e guaspe d'argent daurat ab senyal reyal, e al manech .VI. senyals reyals, ab una corregeta de seda blave, caps e mosqueta e .IIII. platons d'argent ab lo foure d'atzeytoni blau.

[ 9.] Item altre basalart cap e croera guarnit d'argent daurat, e lo bocal e mig e guaspe d'or ab algunes rosetes de esmalt blanch 
e de roigcler, ab la corregeta de sada vermella, ab cap, e fivella, e .V. platons d'aur.

[10.] Item . $I^{\mathrm{a}}$. copa de mavre ab lo peu guarnit d'or e entorn del peu ha flors de lir, a .I. esmalt dedins, ab sobrecop ab esmalt e flors de lir.

[11.] Item .I. saler d'argent daurat ab peu, e cobertor $a b . I^{a}$, brancha de coral. Pesa menys de la branca .I. march, . . ${ }^{\mathrm{a}}$. onza, .III. quarts mig.

[12.] Item . I $^{\mathrm{a}}$. copa petita d'argent daurada e blanch, ab sobrecop.$I^{a}$. pedra de vidre blava e en la copa .I. esmalt ab.I. hom vestit de vert, e al sobrecop .I. altre esmalt blau ab.I. pin vert e.I. sauc, que li esta dipres. Pesa .I. march, .II. onzes, .II. quarts e mig.

[13.] Item .I. estoig de cuyr en que esta .I. pitxer d'argent daurat dins e de fora, e sobrecop de part de dins marcat ab corona. E pesa .II. marchs, .III. onzes e mige.

[14.] Item .I. saler de nou d'India, guarnit d'argent daurat, e al cap ha .I. ausalet ligat ab cadena.

[15.] Item .I. cubell d'argent daurat sobredaurat en que ha .I. senyal e creus a la deredor, ab son sobrecop. E pesa .I. march, .VII. onzes, .III. quarts.

[16.] Item .II. salers de cristall ab ores d'argent, e los peus de fulles d'arbre, en que ha VIIII. perles en cascun peu petites, ab .III. escudets reyals.

[17.] Item .I. parell de bacins d'argent, sobredaurats dins e de fora, de levar mans, en que ha .I. esmalt, es hi lo timbre e.I. senyal reyal baix, e creu de Sent Jordi a la una part e altre. Pesa VIIII. marchs e mig.

[18.] Item .I. verigle ab manech d'argent daurat.

[19.] Item .I. confiter d'argent ab los suatges daurats e lo pom del cano daurat, e dalt .I. esmalt ab senyal reyal, e ab peu ab lo suatge daurat. Pesa .VIII. marchs, .I. onza.

[20.] Item .II. terraces de mavre que entre la una dins l'altre, ab los peus e les ances d'argent daurat, esmaltades les nances, e en la que va de part dessus .I. esmalt blau a .II. papagays.

[21.] Item .I. confiter d'argent daurat esmaltat, ab lo timbre de dins en lo mig, e senyals reyals al peu, e esta en .III. peces. E pesa VI. marchs, .III. onzes, e mige.

[22.] Item .II. curelles (sic) d'argent daurades, ab manech de coral, e al cap de cascuna ha dues broques d'argent daurat.

[23.] Item .I. cana e tres palms de parge de fill d'or e de seda morada.

[24.] Item dues ales de vibra d'argent daurades e esmaltades de blau. Pesan .I. onza e quart.

[25.] Item .I. ganivet ab lo manech d'ambre, d'argent levorat 
ab ambre dintre, e .I. esmalt vert al cap, e lo foure de velut morat, ab .III. virolles d'argent daurades, ab .II. senyals reyals, e cordo de seda vermella, e boto d'aur.

[26.] Item .I. sinyell d'argent daurat a manera de folosents. Pesa .I. march, $I^{\text {a }}$ onza.

[27.] Item .I. reliquiari petit, ab peu, d'argent daurat, ba. III. esmalts al peu, fall-ne la .I., en lo qual ha dels osses e del peno de Sent Jordi, dins un cano de cristall.

[28.] Item .I. altre reliquiari d'argent, daurat e esmaltat, de la una part ha una ymage in Sede Magestate, e de la altre part es una taula de Strologia, ab .I. cordo de fill d'aur e de seda vermella. Lo qual esta en una capceta de fust.

[29.] [Item .I. con]fiter d'argent, tot daurat, e a la part dessus fall l'esmalt, e en lo pom del mig del cano ha VI. esmalts ab rosetes grogues. Pesa .VIIII. marchs, VII. onzes, .I. quart.

[30.] Item .I. altre confiter d'argent al les suatges daurats, son .II. peces, e lo peu baix es levadiç; dessus ha .I. senyal reyal Pesa .III. marchs, VII. onzes, mige.

[31.] Item .I. altre confiter d'argent ab son peu, e alt en lo mig ha.I. esmalt en que ha .I. arbre ab .I. porch, ab lo cano e pom, los suatges daurats, ab .III. esmalts en lo peu. Pesa .VIII. marchs, VII. onzes, mige.

[32.] Item .I. bassi radon d'argent blanch, que servia a la selleta. Lo qual esa .V. marchs, . ${ }^{2}$. onza.

[33.] Item .I. bassi d'argent daurat dins e de fora, ab .I. esmalt ab senyal reyal al mig. Pesa. IIII. marchs, .II. onzes, .I. quart.

[34.] Item $\mathrm{II}^{\mathrm{es}}$ canalobres d'argent blanchs, ab canons en que ha sengles poms, e ab les suages e poms daurats, e ab VI. esmalts en lo pom de cascu, pesa, les quals servien quant fahia la oracio, .IIII. marchs, .V, onzes e mige.

[35.] Item .1111. canalobres d'argent, los .11. se encasten dins los altres . II ${ }^{o s}$. ab los suatges daurats, los qui servien a la oracio. Pesen .V. marchs, .VI. onzes.

[36.] Item .II. girasolls d'argent daurats dins e de fora,. Pesan .I. march, .IIII. onzes, .III. quarts.

Totes les cosas dessus dites foren meses e estojades en .I. coffre encuyrat de cuyr ras, ab raunes de ferre e intitulat en un poch de pergami, Segon de la Cambra, Ab .II. panys, lo .I. dels quals no poth tenquar, e l'altre es tenquat ab clau, e en mig de abdos los panys ha .II. senyals reyals e fou ligat lo dit cofre ab corda de canem, e segellat ab lo segell del anell petit de la Magestat.

[37.] Item .I. reliquiari d'aur ab son peu, en lo qual peu ha VI. esmalts; los .III. a senyal d'Arago, e los .III. a senyal reyal de Comte de Barchinona. Sobre lo dit peu ha .I. cano de cristal 
ab cubertor tot guarnit d'aur. E dins lo cano de cristall es la camisa de Jhesucrist, dessus lo cobertor ha .I. gros balaix e .II. perles grosses, que estan asseguts e encastats sobre .I. pinyo d'aur, lo qual pinyo d'aur ab lo dit .I. balaix e dues perles es levadiç, e sobre'l pe[u] per on se obre lo dit cobertor havia cera vermella, en que era la empremta del anell de la Magestat. Lo qual reliquiari es dins.I. estoig de cuyr negre, e lo dit estoig dins una caxeta de cipres, tencada ab clau.

[38.] Item .I. altre reliquiari d'aur d'obre de fill tirat, en lo peu del qual ha .IIII. esmalts reyals e.II. d'Arago, e sobre lo dit peu ha .II. branques, e en cascuna brancha ha .I. angell d'aur, qui tenen la pinta de Madona Santa Maria, qui es encastada en aur, e ab la pinta ensemps ha dels cabells de Madona Santa Maria, en la qual ha encastats.XXIII. granats e .XXIII. safirs, e de la una part .IIII. turqueses, e de la altre part .IIII. perles grossetes, e en los perns .V. perles petites, estibat en.$^{2}$. capça de fust pocha, ab coto, e una tovallola deaseda ab flocadura d'aur e de seda.

[39.] Item unes taules de vori encastades en argent daurat, esmaltat de la part de fora, e son dues taules plegadisses; e de la .$I^{2}$. part ha figurades.$I^{\mathrm{es}}$. figures de reys, e de la altre part es en esmalt la figura de Madona Santa Maria ab lo Jhesus, feta a semblança que seu en . $I^{a}$. cadira; figures de Verge e de Sent Johan qui li esta entorn. E sobre les dites figures ha .IIII. figures d'angels petits, e de la part de dins ha moltes e diverses istories e figures de vori, qui son de la Passio de Jhesucrist e d'altres diverses Sants. Les quals taules son enbolcades en.I, troç de sendat vermell, e conservades en .I. caxo de cuyr cruu negre.

[40.] Item .I. peu d'argent daurat ab .III. esmalts, fets, ço es, los .II ${ }^{\mathrm{os}}$. a manera de creu e los .II. a tres migis compas ab figures de baboynis, e en lo dit peu ha .I. cano d'argent, semblantment daurat e siellat, lo qual serveix e es fet per tenir lo Sant Fust Major de la Santa Veracreu, qui era del dit Senyor. Lo qual pesa .V. marchs e mig, e es conservat lo dit peu en .I. estoig de cuyr cruu, laonat de ferre.

[41.] Item .II. reliquiaris d'aur ab peu e ab cano, en lo mig dels quals ha en cascu .I. pom fet a .VI. cayres, e ab cap de cascun peu ha .I. cano de cristall, dins cascun del qual es.$I^{2}$. de les espines de la Corona de Jhesucrist. E sobre cascun dels dits canons de cristal ha .I. capitell d'aur. E sobre cascun capitell esta una creu petita. Los quals reliquiaris son conservats en sengles estoigs de cuir vermell.

[42.] Item una creu d'aur, qui penja en .I. cordo de seda morada, dins la qual ha encastat del Fust de la Santa Veracreu, e altres diverses reliquies de Jhesucrist, e de la part davant de la dita creu, ço es, en lo mig, ha encastat un balaix, e entorn 
d'aquell III perles e en los braces de la dita creu ha encastades .IIII. puntes de diamants, e a la punta de cascun braç de la dita creu .IIII. perles, e al mig de cascun quaern de perles ha encastat .I. diamant petit. La qual creu es conservada en .I. estoig de cuyr cruu; lo qual estoig esta dins .I. cofret encuyrat, ferrat ab sa tencadura. E pesa la dita creu ab les coses dessus dites .IIII. onzes, e VI argenç.

[43.] Item fou atrobat dins lo dit cofret .I. reliquiari d'argent, fet a manera de foguer, daurat de la part de fora e per les vores, e al mig del reliquiari ha .I. esmalt ab senyal de creu de Sent Jordi, dins lo qual ha diverses reliquies de Sants. E pesa, ab tot ço que dit es, .IIII. onzes, e mige e .I. argenc.

[44.] Item fou atrobat dins lo dit cofret $\mathrm{I}^{2}$. bossa de drap de seda vermell, ligada ab seda vert, dins lo qual ha segons que.s diu diverses reliquies.

[45.] Item .I. trocet de cuyr de cervo, dins lo qual ha ligat dins un paper de la Pedre del Monument de Jhesucrist, e.I. petit de la Verga d'Aron.

[46.] Item hi fo atrobada. $I^{a}$. carta de dispensacio del matrimoni del senyor rey en Johan e de la senyora Reyna Dona Yolant.

$\mathrm{E}$ totes les dites coses foren meses e conservades dins.I. coffre de cipres, encuyrat de cuir ras, e laonat de ferrre, ab sa tencadura; lo qual fou segellat ab lo segell del anell de la Magestat e ligat o troçat ab una corda de canem.

[47.] Item una creu d'aur, en la qual de la una part ha encastat una creu del Sant Fust de la Veracreu, e ha encastats .XII. balaix, .VIII. maragdes, .XLII. perles grosses, e de la altre part ha .X. esmalts, ço es, .I. gran en mig de la creu ab figura de Sede Magestatis, e ha en cascun cap .I. dels .IIII. Evangelistes, e los romanents .V. verts d'esmalt clar. La qual pesa ab tot ço que dit es, la qual es conservada dins .I. estoig de cuyr cruu negre, .VIII. marchs, VII. onzes III quarts.

[48.] Item una capça de vori ab cobertor e tancadura qui.s tanqua ab tornet, guarnida e laonada de leuto; e en lo dit cobertor ha .I. esmalt de lauto, al mig del qual ha .I. granat, e .VIII. maragdes, pents uuls de rata. $\mathrm{E}$ dins la dita capça ha .III. didals de vidre; la .I. dels es enserpellat de drap d'aur buyt, e en los romanents dos ha sengles albarans, en cascun dels quals ha escrit Oleum Beate Marie Virginis de Sardenia. E mes avant ha en la dita capça.$I^{\text {a }}$. lengua de serp.

[49.] Item.$I^{x}$. capça ab son cobertor de fust, ligada ab fill d'ampalomar, ab creu, en lo qual cobertor ha escrit de la part dessus Açi ha de la ossa de Sent Johan, de Sent Steve, de Sent Jordi e de Santa Barbara.

[50.] Item .II. saquets ligats, ço es, la un de sendat vert, ligat 
ab fill de li blanch, e l'altre de lens prim, ligat ab seda groga, en que appar que hage reliquies.

[51.] Item .I. caxonet de fust de cipres ab son cubertor levorat e laonat de diverses marquets, dins la qual ha diverses padassets de drap de seda, en que ha diverses osses de Sants, cascun intitulat de quals Sants son.

[52.] Item.$^{\mathrm{a}}$. caxeta petita ab son cobertor, guarnida de fulla d'estany, e en lo dit cubertor ha algunes figures fetes a compas, e entorn figures de homens, dins la qual ha alguns pedassets de sendat e papers, que par que sien de reliquies.

[53.] Item .I. saquet petit de sendat vermell, ligat ab fill de li blanch, dins lo qual ha diverses ligams de papers, en que appar que hage algunes reliquies de Sants.

[54.] Item . $I^{x}$. capceta de fust ab son cubertor pintada, dins la qual ha .I. troç de drap blanch, albaranat, en que ha escrit De la vestedura de Sant Pere de Morrona.

$\mathrm{E}$ totes les dites coses foren atrobades dins .I. mig coffre, quayx cayrat, encuyrat de cuyr ras, e laonat de ferre, dins lo qual coffre ha una caxeta de cipres ab sa tencadura, e guarnida per les cantons de lauto, dins la qual la dita Veracreu e altres coses dessus dites son conservades. $\mathrm{E}$ lo dit coffre fou tencat ab clau, e ligat ab corda de canem, e segellat ab lo segell del anell de la Magestat.

Item .1. altre mig coffre semblant del dessus dit, encuyrat e laonat de ferre, ab sa tencadura, dins lo qual foren atrobades les coses següents:

[55.] Item .I. peu d'argent ab cano tot daurat, en lo qual ha .IIII. esmalts, ço es, en lo dit peu, lo qual serveix e esta una creu d'aur. Lo qual dit peu pesa .II. marchs, .V. onzes, .II. quarts e mig. Lo qual peu es conservat dins .I. estoig de cuyr cruu negre.

[56.] Item .I I $^{\mathrm{a}}$. creu d'aur, qui es feta per estar lo dit peu, dins lo qual ha conservat del Fust de la Santa Veracreu, e segellat la on se obre ab cera vermella; en la qual ha .IIII. safirs grosses, ço es, un en cascun cap de la dita creu, e .V. safirs menors dels dessus dits, e VIII. balaix, e en lo mig ha .I. maragde gran, e IIII. perles grosses, e a cascun cap de la dita creu .I. tern de perles migenceres, e deins lo dit maragde .I. quaren de perles migenceres. La qual pesa ab totes les dites coses .I. march, .II. onzes e.III. quarts. La qual creu es conservada dins un estoig de cuyr negre; la qual creu e peu son conservats dins un caxo ab son cubertor de cipres e ab tencadura, e guarnida en los cantos de laones de ferre.

[57.] Item. $I^{a}$, capceta ab cobertor de fust, dins la qual es conservada una carta ab segell pendent de cera vert, testimonial del Fust de la Santa Veracreu. 
[58.] Item unes taules de pint plegadisses, que son .IIII. peces, de pint de Romania, en les quals son figures del Crucifix de Jhesucrist, e de Madona Santa Maria e d'altres diverses Sants.

[59.] Item unes altres taules pintades, qui son dues peces plegadisses de pint de Sena, en la una de les quals es la figura del Crucifix de Jhesucrist e dels dos ladres. E en l'altre de la Resurreccio.

[60.] Item altres dues taules pintades d'obra de Sena, plegadices, en la una de las quals es la figura de Madona Santa Maria ab l'Infant Jhesus e en l'altre dos angells.

[61.] Item altres dues taules plegadices, pintades d'obra de Sene, en la una de las quals es la figura de Madona Santa Maria stant en oracio; en l'altre de la figura de Sant Gabriel.

E les tres taules prop dites, son conservades de dos en dos, en sengles stoich de cuyr vermell. E totes les dites coses foren tornades e conservades en lo dit cofre, lo qual era ab peus alts de ferre, e tancat ab clau, e ligat ab corda de canem, e segellat ab lo dit segell.

[62.] Item .I. reliquiari d'argent daurat, ab peu, en lo qual ha quatre esmarts, ço es, los dos a senyal reyal, e los altres dos ab senyal del Rey de Xipre; sobre'l qual peu ha un braz d'argent daurat, que ha figura d'un cavaller, ab escut, ab senyal de Sent Jordi, e ab lanze en la ma, stant sobre un cavall e fer una vibra; e es daurat tro a la ma, la qual ma es blanca, senyant, dins la qual ha del braz de Sant Jordi. Lo qual ab tot ço que es dins, pesa .XII. marchs, .VI. onzes. E lo qual es conservat en un stoich de cuyr vermell.

[63.] Item un altre reliquiari d'aur, fet a .VI. cayres, e ab un cano alt, en lo mig del qual ha un pom; e al cap del dit cano ha . II $^{\mathrm{s}}$ pezes de cristall, encastades dins una roda d'aur, ab diverses letres fetes a la redor, dins la qual roda son conservats corporals. E pesa, ab ço que dit es, quatre marchs, una onza, .I. quart. E lo qual es conservat en un stoich de cuyr negre. E totes le dites coses foren tornades e conservades en un cofre vey encuyrat de cuyr ras e laonat de launes de ferre, lo qual fou tancat e ligat ab corda de canem, e segellat, ab lo dit segell.

[64.] Item una taula de fust, guarnida per les vores a la redor de fulla d'argent daurat, ab esmalts d'argent, ab senyal del Rey de Cicilia. E en la faz dintre de la dita taula ha diverses figures de homens, de dones, e de cavallers, e sobre les dites figures ha diverses peces de cristall, fermats o sitiats en colondells de jaspi. E en la un cap de la dita taula ha un taulell d'escats de la dita obra. E en la partida detras es cuberta de fulla d'argent fort prima.

[65.] Item quatre peus d'argent ab sengles figures de leons al 
cap de cascun, sobre los quals esta la dita taula. E es cert que los dits peus de dintre son de fust, per la qual rao no foren posats.

[66.] Item una caxeta feta a cayre de fust, dins la qual ha e es conservada una gran brancha de coral. La qual taula e altres coses dessus dites son conservades dins una caxa de fust vella, en que ha pintats senyals del Rey de Cicilia, la qual fou tancada e segellada ab lo dit segell.

[67.] Item .IIII. spases morisques de la gineta, guarnides d'aur, ço es, los poms, mantins e croeres, e en cascuna de les quals ha tres bocals, e guaspes d'aur, e corregetes de seda de diverses colors, ab mosquetes, caps, fivelles e platons tot d'aur esmaltat.

[68.] Item una altra spasa armeniença o turquesa, ab lo manti de camut gornit d'argent daurat, e en lo foure ha .IIII. bocals, e guaspa, e un smalt d'argent daurat, e ab correja ab lo parge de seda blava, ab fivelles, mosquetes, e platons, e cap d'argent daurat.

[69.] Item un estoig de fust per tenir vires, ab tancadura e clau d'argent, ab .III. panys d'argent, dins lo qual ha diverses vires.

[70.] Item .III. parells de patins de cuir morischs.

[71.] Item una vestimenta feta a manera d'aljuba, qui son IIII. peçes, ço es, la sobiran de drap de seda groch, l'altra de boqueram blanch, l'altra de drap de seda, listat de diverses colors, folrat de tela blanca, e l'altra de boqueram blanch.

[72.] Item. I. altre vestimenta feta a manera d'aljuba, qui son IIII. peces o vestiments, ço es, la primera de drap de seda blanch; la segona de boqueram blanch; la . III $^{\mathrm{a}}$. de drap de seda de diverses colors ab letres morisques, folrada de tela blancha; e la quarta de boqueram.

[73.] Item una alcandora curta de tela de li prim, ab sengles barres en cascuna manega de seda negra.

[74.] Item .I. alfilem de drap de li blanch, prim, ab les ores de seda blava, ab .I. listonet al mig de seda morada, fet per portar al cap quant cavalquen e juguen a la gineta.

[75.] Item una aljuba de dos draps de cotonina prima, tots blanchs, laborat d'obra d'agulla.

[76.] Item .I. tinter de fust ab diverses marquets, d'óbra de domas ab tallapenes e tisores dins.

E totes les dites coses foren meses e conservades en .I. coffre cubert de cuyr ras ab dues tancadures, launat de ferre, e dessus ab senyals del Regne de Mallorques, lo qual fou tancat ab clau, e ligat ab corda de canem, e segellat ab lo segell dessus dit.

[77.] Item una colla gran d'argent blanch ab dues anses. Qui pesa .XV. marchs, .II. onzes.

[78.] Item altra olla d'argent menor de la prop dita ab .IIII. 
anses, e ab cobertora, d'argent blanch. Qui pesa .VIII. marchs, .IIII. onzes.

[79.] Item una altra olla d'argent blanch, menor de la prop dita, ab dues anses. Qui pesa .V. marchs, una onza.

[80.] Item una caçola d'argent blanch ab dues anses, qui pesa .III. marchs, .V. onzes e mige.

[81.] Item una loça d'argent blanch ab son ganxet. Qui pesa .II. marchs, .VI. onzes.

[82.] Item una bromadora d'argent blanch ab son ganxet. Qui pesa .II. marchs, .VI. onzes.

[83.] Item una olleta de coure petita ab dues ançes.

[84.] Item avia dins un cofre encuyrat de cuir ras, enlaunat de ferre, e dins lo cofre havia una caxeta de cipres, ab son cubertor corrediz, e tancadura, e guarnida de ferre per los cantons, dins la qual avia, primerament, una ymage d'argent feta a figura de Sant Steve qui sta sobre .I. peu d'argent; lo qual peu e Ymage es tot daurat; e te la dita ymage en la ma [una] palma d'argent smaltad d'esmalt vert, e en la man sinistre un libre e un maniple d'argent daurat; e la vestedura que porta es feta a manera de dalmatica e te en lo pits un senyal reyal, ab corona; dessus e sobre la corona un smalt blau. La qual pesa .XV. marchs, . $I^{\mathrm{a}}$. onza.

[85.] Item dos canalobres d'argent blanch, cascun ab tres peus, e ab cano, al mig de cascun dels quals ha un pom daurat ab.VI. smalts en cascun, e en lo peu de cascun a tres smalts a senyal reyal; e sobre lo dit cano ha una copa d'argent, e al mig de cascuna copa . . I'. broqua d'argent, e ab los susages de cascun daurats. E es certs que del pom del pom (sic) del un dels dits canalobres fall un smalt. Qui pesan .VI. marchs, .VII. onzes.

[86.] Item un reliquiari d'argent daurat $a b$ peu, fet ab quatre compassos, e ab cano sobre lo qual ha un tabernacle o oratori d'argent daurat, ab portes qui.s obren, tot daurat e muronat; e sobre'l dit tabernacle ha un cimbori, en la cima del qual ha un pinyo, tot daurat, e dins lo dit tabernacle ha una costella de Santa. E pesa ab tot tres marchs, una onza, miga. Lo qual es conservat en un stoig de cuir cruu vermell.

[87.] Item tres taules d'argent smaltades, fetes per donar pau, ab les vores dauradas, e ab ança d'argent en cascuna; en la una de les quals es la figura del Crucifix, ab smalt vert e blau, e es tota daurada de la part detras; e les romanents dues a en cascuna figura de Crucifix, de Santa Maria, e de Sant Johan, ab lo camp d'esmalt blau, vert e morat, e no son daurades de la part detras. E pesen entre totes III marchs e II onzes.

[88.] Item VI smalts o bronges d'argent daurades e smaltades de la part de fora de diverses figures de Jhesucrist, de Sants e de Santes, en les quals ha, ço es, en les .V. en cascuna dos tim- 
bres del senyor Rey e.II. scudets a senyal reyal; e en la romanent una ha quatre scuts fets a senyal reyal, ab .I. fiblall d'argent blanch, la qual es desclavat. Los quals pesen .V. marchs, dos onzas.

[89.] Item .I. caliz ab peu, en lo qual ha VI smalts a senyal reyal, e ab cano ciellat e muronat e al mig a un pom ab. VI. smalts ab senyal del dit senyor; e hay patena, e al mig de la qual ha un smalt a figura de Jhesus in Sede Magestatis, e en les vores ha .IIII. smalts a figura des .IIII. Evangelistes; lo qual calçe e patena, ab tot co que dit es, es tot d'aur. E pesa.VI. marchs, I. quart, mig. Los quals calze e patena son conservats en un estoig de cuir vermell, en que ha senyals reyals.

[90.] Item un calze ab patena d'argent daurat e smaltat, lo peu del qual es fet a VI. puntes, ab .III, esmalts; lo .I. a senyal del Crucifix e de Santa Maria, e de Sent Johan; e l'altre de del Jhesus, e de Santa Maria, que seen in Sede Magestatis; e en l'altre la Salutacio de Santa Maria. E en lo dit calze ha cano, en mig del qual ha .I. p[...] ab .VI. esmalts; los .III. en que ha en cascu .I. testa de .I. Sant, e en los romanents .III., sengles senyals reyals. E lo dit cano ha .I. esmalt sobre lo qual seu la copa del dit calze ab .VI. fulles en cascuna de les quals ha figures de Sants. E en lo mig de la dita patena ha .I. esmalt blau ab la figura de Jhesus, e entorn de la dita patena ha.IIII. esmalts a figura dels quatre Evangelistes. Los quals dits calçe e patena son daurats dins e de fora. $\mathrm{E}$ pesa quatre marchs.

Totes les coses damunt dites, ab les dites olles, caçola, loça e bromadora, exceptat la olla d'argent major, e la olla petita de coure, foren meses e conservades dins lo dit cofre, lo qual fou tencat ab clau e ligat ab tronyella, e segellat ab lo segell del anell de la magestat.

[91.] Item . I $^{\mathrm{a}}$. testa d'argent ab .I. cercle daurat entorn del coll, en lo qual ha .III. esmalts, ço es, .I. en cascun muscle en que es lo timbre e lo senyal del senyor Rey en .I. scut, e devant en lo pits .I. senyal de Sent Jordi, e .I. titol de letres entorn del dit cercle, qui diu Sanctus Ruralius cognatus Regis Spanie de societate. $X I^{m}$. Virginum; e los cabells de la barba e del cap daurats, en la qual ha de la testa del Sant. Qui pesa .XV. marchs, $I^{2}$. onza.

[92.] Item .I. reliquiari tot d'argent daurat, ab peu qui.s soste sobre VI. grius, e es tot ciellat; e sobre to dit peu ha .I. cano alt obrat de cimboris e muronat ab esmalts blaus; e del dit cimbori ixen . II $^{\mathrm{es}}$. branques en cascuna de les quals sta .I. angell qui tenen .I. reliquiari de cristall, fet a manera de cano, e los caps obrats d'argent daurat; e en cascun cap ha .I. esmalt, la .I. a figura de Madona Santa Maria ab l'Infant Jhesus, e l'altre de Sede Majestatis. E de dins lo dit cano de cristall se demostra 
o ha scrit .I. titol, De lapide calumpno ad quam Dominus noster Jhesus Cristus alligatus et flagellatus, etc.. E lo dit cano de cristall se tanca ab.I. losa d'argent, lavorada e daurada del dit obratge; e sobre la dita tancadura ha. .I. cimbori sobre'l qual ha una creu de figura de Crucifix; e a cascuna part del dit cimbori ha .I. pilaret en que stan, ço es, en la .I. la Maria, e en l'altre Sent Johan. Qui pesa .X. marchs, .IIII. onzes. Lo qual es conservat dins .I. estoig de cuir cruu negre.

[93.] Item una corona d'argent, daurada, feta per servir a I a ymage de Madona Santa Maria, ab .I. vel de poca valor; en lo cercle de la qual ha .VII. perles, e .V. pedres de poca valor, les tres verdes e les dues vermelles; e sobre lo dit cercle ha .VIIII. flors, fetes a manera de flors de lir, en les quals ha entre totes XXXVIII perles e .XXXVIIII. pedres de vidre entre vermelles, verdes e blaves. La qual pesa ab les dites coses VI. onzes, .II. quarts e mig.

[94.] Item .I. caxeta petita de vori ab sa tancadura, e launada de lauto, e ab sa clau, dins la qual es conservada e esta.$^{\mathrm{a}}$. caxeta d'argen ab les vores daurades, e ab tancadura feta a manera de sobrepany, e de la part dessus feta tota a senyal real, ab.II. tires de letres en .I. campet blau, la .I. que diu, Crisma Oleum, etc, e l'altre, sal, etc., e ab I anseta d'argent daurada. La qual caxeta d'argent pesa axi com sta .I. march, .II. onzes, .III. quarts.

[95.] Item una taula feta d'obra de musica de diverses istories e figures embotides, la qual es guarnida d'argent, de la part deins blanch, e per los costats, e de la part dessus daurat; ab .I. anell fet a IIII mig compases, d'argent blanch, de la qual no fall res. La qual no fo pesada, e es conservada dins. I. caxonet de cipres ab son cubertor.

[96.] Item una figura de de Sent Rayner, tota d'argent daurat, ab peu fet a diverses finestratges, en lo qual ha entorn .VI. esmalts f[fets] a senyal real, sobre lo qual peu sta la dita figura ab bordo d'argent que te en la man dreta, e en la sinistra .I. llibre, e al cap .I. capell de sol d'argent daurat, ab los cordons d'argent; e es cert que los braços e mans de la dita figura dessoldades, e la meytat del boto del cap del bordo es desoldat. E pesa .XVII. marchs, II $^{\text {es }}$ onzes, mige.

[97.] Item .I. peu d'argent daurat fet a .VIII. cayres, sobre'l qual ha .I. braç d'argent ab botonadura, tot daurat, e al peu del braç ha .I. esmalt blau a figura de Sent Jordi, e al cap del braç ha una ma d'argent blanch qui senya, en la qual a la part de fora ha .I. rexat d'argent d'on se poden veure reliquies de Sent Jordi, qui son dintre la dita ma; e en lo dit major que la dita ma ha.I. rexat d'argent per hon se vehen reliquies qui son dins 
lo dit dit. Lo qual peu e braç, e ma pesa, ab tot ço que dit es, .III. marchs, VII. onzes.

[98.] Item .I. altre peu d'argent daurat, fet a .VI. compasses, en to qual ha .III. esmalts, ço es, lo.I. a figura de Sent Nicolau ab mitra al cap, e croça en la ma e los dos a senyal real; e sobre lo dit peu ha .I. braç qui ix del mig de VI. capitells, muronats e esmaltats, e en cascun capitell ha .II. esmalts a senyal real. E lo dit braç es rexat per veure les reliquies de Sent Nicholau, qui son dins lo dit braç; ha $\mathrm{I}^{\mathrm{a}}$ ma d'argent blanch, quì senya. Lo qual pesa .VI. marchs, VI. onzes, miga.

[99.] Item .I. altre peu d'argent daurat, fet a .VI. cayres, sobre'] qual ha $I^{\mathrm{a}}$ garlandeta, de la qual ix .I, braç d'argent daurat, e botonat, e al cap del braç ha $I^{\mathrm{a}}$ ma d'argent blanch, que senya, e en lo dit major ha rexat d'argent, per on veu hom reliquies de la ossa de Santa Barbara, que ha en lo dit dit. E pesa .V. marchs, .I. quart.

E totes les dites coses, ensemps ab la olla major d'argent, e la petita de coure e VI. sobrepellicets de drap de li, e . I ${ }^{\mathrm{a}}$. lina de drap de li de altar, foren meses e conservades dins .I. cofre encuyrat de cuyr ras, e launat de launes de ferre, ab sa tancadura, e segellat ab lo segell del anell de la Majestat, e ligat ab tronyella d'espart." 


\section{ÍNDEX}

\section{A}

afiblall 8

agulla, obra d' 75

albarà 48

alcandora 73

alfilem 74

aljuba $71 ; 72 ; 75$

ambre 25

àngel $38 ; 92$

arbre 31

argent $3 ; 8 ; 16 ; 19 ; 25 ; 30 ; 31$; $35 ; 43 ; 55 ; 64 ; 65: 69 ; 78 ; 84$;

$85 ; 87 ; 91 ; 94 ; 96 ; 97$

-blanc $3 ; 4 ; 12 ; 32 ; 34 ; 77$;

$78 ; 79 ; 80 ; 81 ; 82 ; 85$;

$88 ; 95 ; 97 ; 98 ; 99$

-daurat $1 ; 2 ; 3 ; 8 ; 9 ; 11$;

$12 ; 13 ; 14 ; 15 ; 17 ; 18 ; 19$;

$20 ; 22 ; 25 ; 26 ; 27 ; 29$;

$30 ; 31 ; 33 ; 34 ; 35 ; 36$;

$39 ; 40 ; 43 ; 55 ; 62 ; 63$;

$68 ; 84 ; 85 ; 86 ; 87 ; 92$;

$93 ; 94 ; 95 ; 96 ; 97 ; 98 ; 99$

-daurat i esmaltat 21; 24;

$28 ; 39 ; 88 ; 90$

- esmaltat $84 ; 87$

atzeitoní 8

B

bací $32 ; 33$

- per fer la barba 4

- per levar mans 17 balaix $37 ; 42 ; 47 ; 56$

basalart $6 ; 7 ; 8 ; 9$

bocaram $71 ; 72$

bordó 96

bossa 44

braç $97 ; 98 ; 99$

bromadora 82

C

caixa, caixeta, caixó, caixonet

$37 ; 39 ; 51 ; 52 ; 54 ; 56 ; 66 ; 84$;

94

caldereta 3

calze $89 ; 90$

canelobre $34 ; 35 ; 85$

cànem, corda de $36 ; 46 ; 54$;

$61 ; 63 ; 76$

canó 27

capell de sol 96

capsa, capseta $38 ; 48 ; 49 ; 54$;

57

carta

— de dispensació del matrimoni del rei Joan i de la reina Violant 46

- ab segell pendent de cera verd 57

cassola 80

cavall 62

cinyell 26

cofre, cofret $36 ; 42 ; 46 ; 54$ :

$63 ; 76 ; 84: 99$

confiter $1 ; 19 ; 21 ; 29 ; 30 ; 31$ 
copa $10 ; 12$

coral 22

-branca de 11; 66

corda $36 ; 46 ; 54 ; 61 ; 63: 76$

cordó $25 ; 28 ; 42$

corona $1 ; 93$

-marcat ab 13

corporal 63

corretja, corretgeta $5 ; 6 ; 8 ; 9$;

$67 ; 68$

cotó 38

cotonina 75

coure 83

creu $15 ; 40 ; 41 ; 42 ; 47 ; 49 ; 55$;

56

-de figura de Crucifix 92

-de Sant Jordi $17 ; 43$

cristall $16 ; 27 ; 37 ; 41 ; 63 ; 64$;

92

crossa 98

cubell 15

cuir $7 ; 13 ; 37 ; 41 ; 56 ; 61 ; 63$;

$70 ; 89$

- de cervo 45

-cru $39 ; 40 ; 42 ; 47 ; 55$; $86 ; 92$

-ras $36 ; 46 ; 54 ; 63 ; 76 ; 84$; 99

culleres 22

D

dalmàtica 84

diamant 42

-punta de 42

didal 48

domàs 76 drap

-albaranat 54

-d'or 48

\section{E}

empresa de la corona (; 7

encuirat $36 ; 42 ; 46 ; 54 ; 63 ; 76$;

$84 ; 99$

escut, escudet

-reial $16 ; 88 ; 91$

-de Sant Jordi 62

esmalt $1 ; 9 ; 10 ; 12 ; 17 ; 19 ; 20$;

$24 ; 25 ; 27 ; 29 ; 31 ; 33 ; 34 ; 37$;

$38 ; 39 ; 40 ; 43: 47 ; 48 ; 55 ; 62$;

$68 ; 84 ; 85 ; 87 ; 88 ; 89 ; 90 ; 91$;

92; 96: 97; 98

espart 99

espasa

- armeniença o turquesa 68

-morisca 67

estoig $13 ; 37 ; 40 ; 41 ; 42 ; 47$; $55 ; 56 ; 61 ; 62 ; 63 ; 69 ; 86: 89$;

92

ferro $6 ; 36 ; 40 ; 42 ; 46 ; 54 ; 56$;

$61 ; 63 ; 76 ; 84 ; 99$

figura 95

-àngel $39 ; 60$

-baboí 39; 40

- cavaller 64

-Crucifix de Jesucrist 58; 87

-Crucifix de Jesucrist i dels dos lladres 59 
-Crucifix, Santa Maria i Sant Joan 90

-dones 64

- feta a compàs 52

-històries 39; 95

-homes 52; 64

- Jesús 90

- Jesús in Sede Magestatis $89 ; 47 ; 92$

-Jesús i Santa Maria in Sede Magestatis 90

-Jesucrist 88

-lleons 65

-Madona Santa Maria $58 ; 87$

- Madona Santa Maria ab lo Jesús $39 ; 60 ; 92$

-Madona Santa Maria estant en oració 61

-Passió de Jesucrist 39

-Quatre Evangelistes 47; $89 ; 90$

-reis 39

-Resurrecció 59

- Salutació de Madona Santa Maria 90

-Sant Gabriel 61

-Sant Joan 87

-Sant Jordi 62; 97

-Sant Nicolau 98

-Sants i Santes $39 ; 58 ; 88$; 90

-testa de Sant 90

-Verge i Sant Joan 39 fil de

-empalomar 49

- or e de seda $23 ; 28$ -tirat 38

flor de lliri $10 ; 93$

foure $6 ; 8 ; 25 ; 68$

fulla 90

-d'arbre 16

fusta $28 ; 38 ; 49 ; 51 ; 54 ; 57$;

$64 ; 65 ; 66 ; 69 ; 76$

G

ganivet $7 ; 25$

- fet a manera de basalart 6

garlanda, garlandeta 99

-de fulles 1

girasol 36

granat $38 ; 48$

griu 92

hom vestit de vert 12

I

imatge de

-Sant Esteve 84

- Sant Joan 92

-Sant Jordi 62

-Sant Rainer 96

-testa de Sant «Ruralius, cognatus regis Spanie, de societate undecim milia Virginum» 91

-Santa Maria 92

-Sede Magestatis 28 
- crisma 94

olla $77 ; 78 ; 79 ; 83$

jaspi 64

or $5 ; 6 ; 7 ; 9 ; 10 ; 25 ; 37 ; 38$;

$41 ; 42 ; 47 ; 55: 56 ; 63 ; 67 ; 89$

LI

-esmaltat 67

llança 62

llautó $7 ; 48 ; 54 ; 94$

-daurat 6

llengua de serp 48

llenç 50

lletres morisques 72

Ili

-drap de $73 ; 74 ; 99$

- fil de $50 ; 53 ; 56$

llibre $84 ; 96$

lloça 81

M

palma 84

papaga 20

paper $45 ; 52 ; 53$

patena $89 ; 90$

patins moriscs 70

pergamí 36

perla $16 ; 37 ; 38 ; 42: 47 ; 56 ; 93$

peu $2 ; 55: 65 ; 84: 97 ; 98 ; 99$

pi 12

pitxer 13

porc 31

mà $97 ; 98 ; 99$

maçoneria, obrat de 1

maniple 84

maragda $47,48,56$

marbre 10;20

mitra 98

$\mathrm{R}$

reliquia $44 ; 50 ; 52$

- cabells de Madona Santa

Maria 38

-camisa de Jesucrist 37

- costella de santa 86

-espina de la corona de Jesucrist 41

nou d'Índia 14

-Jesucrist 42

- Ossa de

\section{O}

-Sant Esteve 49

-Sant Jordi 27; 49

ocellet («ausalet») 14

oli

-Santa Bárbara 49; 99

-Sants 51

- pedra de Cerdenya 48 
— columna «ad quam dominus noster Jhesucristus alligatus et falgellatus, etc.»

-monument de Jesucrist 45

—pendó de Sant Jordi 27

- pinta de Madona Santa Maria 38

-Sant Fust de la Veracreu $40 ; 42 ; 47 ; 56 ; 57$

-Sant Jordi 62; 97

-Sant Nicolau 98

-Sants 43; 53

-Verga d'Aron 45

- Vestimenta de Sant Pere de Morrone 54

reliquiari $27 ; 28 ; 37 ; 38 ; 41$;

$43 ; 62 ; 63 ; 86 ; 92$

roseta $9 ; 29$

S

safir $38 ; 56$

sal 94

saler $11 ; 14 ; 16$

salpasser 3

salze («sauç) 12

saquet $50 ; 53$

savastre 6

seda $5 ; 7 ; 8 ; 9 ; 23 ; 25: 42 ; 44$ :

$50 ; 51 ; 67 ; 68 ; 71 ; 72 ; 73 ; 74$

senyal 15

-Aragó 1; 37; 38

- comte de Barcelona 37

-reial $1 ; 8 ; 17 ; 19 ; 21 ; 25$;
30 ; $33 ; 36 ; 38 ; 62 ; 84$;

85 ; $88 ; 89 ; 90$; 91; 94;

96; 98

-regne de Mallorca 76

-rei de Sicília 64; 66

-rei de Xipre 62

- creu de Sant Jordi ab quatre testes de moro 1

-Sant Jordi 91

sobrecopa $2 ; 12 ; 13 ; 15$

sobrepellisset 99

tallapenes 76

taula 64

-Astrologia 28

- obra de música 95

- per donar pau 87

- plegadissa 39

- de Romania 58

-de Siena 59; 60

-de Siena 61

taulell d'escacs 64

terrassa 20

timbre reial $1 ; 17 ; 21 ; 88 ; 91$

tinter 76

tisores 76

tovallola 38

tronyella $90 ; 99$

turquesa 38

ulls de rata 48 
vellut 25

vericle 18

vibra $62 ; 64$

vidre $12 ; 48 ; 93$

vira 69

vori $39 ; 48 ; 94$ xiprer $37 ; 46 ; 51 ; 54 ; 56 ; 84$;

95 